\title{
Autor, Lehrer, Wissenschaftler
}

HOLM THIEME Er ist physiopraxis-Autor der ersten Stunde und trotz seines Namens nicht mit dem physiopraxis-Verlag verwandt. Jetzt hat der Physiotherapeut und Neuroreha-Experte Holm Thieme in Halle promoviert und für seine Arbeit sogar einen Preis gewonnen.

Wirst du oft gefragt, ob du mit dem Thieme Verlag verwandt bist?

Ja (lacht). Immer, wenn ich in einem physiotherapeutischen Kontext tätig bin, werde ich danach gefragt. Woher unser Familienname genau stammt und welche Bedeutung er hat, weiß ich allerdings nicht.

Du bist ein Autor der ersten Stunde bei physiopraxis. Wie hat sich die Zeitschrift deiner Meinung nach seit ihren Anfängen entwickelt?

Das Erscheinungsbild und die inhaltliche Gestaltung der Texte sind professioneller geworden. Die Artikel enthalten mehr geballtes Wissen. Mich freut, dass die kritische Auseinandersetzung mit der physiotherapeutischen Arbeit zugenommen hat und Fragen der Evidenzbasierung eine immer größere Rolle spielen. 


\section{Wie effektiv ist Spiegeltherapie nach Schlaganfall?}

\section{Holm Thieme ...}

... ist 33 Jahre alt und wohnt mit seiner Lebensgefährtin und seinen beiden Kindern in Freital bei Dresden. Nach seiner Ausbildung zum Physiotherapeuten arbeitete er in der neurologischen Rehabilitation, wo er unter anderem die Spiegeltherapie kennenlernte. Parallel studierte er Physiotherapie in Hildesheim, wirkte dort in Forschungsprojekten mit und schloss 2005 mit einem Bachelor und 2008 mit einem Master ab. Heute ist er in Hildesheim wissenschaftlicher Mitarbeiter und Lehrbeauftragter. Zudem unterrichtet Holm Thieme Schüler an seiner alten Schule in Kreischa. Nach seinem Studium fand er an der Medizinischen Fakultät der Uni Halle-Wittenberg Unterstützung für sein Promotionsprojekt: die Effektivität der Spiegeltherapie. Seit 2012 ist er promovierter Physiotherapeut. Anfang 2013 wurde seine Doktorarbeit beim Rehawissenschaftlichen Kolloquium der Deutschen Rentenversicherung mit dem Förderpreis für Rehabilitationswissenschaften der Celenus-Kliniken ausgezeichnet.

physiopraxis-Lesern ist Holm Thieme unter seinem Autorenkürzel „hoth“ bekannt, das seit den ersten Ausgaben regelmäßig unter seinen Artikeln in der Rubrik „Internationale Studienergebnisse“ zu finden ist.

\section{Die Dissertation}

Schätzungen zufolge erleiden in Deutschland pro Jahr etwa 150.000 bis 200.000 Menschen einen Schlaganfall. Etwa 70-80\% von ihnen behalten motorische Einschränkungen der oberen Extremität zurück. Um die Armfunktion zu verbessern, kommt seit einigen Jahren die Spiegeltherapie zum Einsatz. Dabei wird ein Spiegel in der Sagittalebene zwischen beiden Armen des Patienten so platziert, dass sich die nicht betroffene Seite darin spiegelt. Nun bewegt der Patient seinen nicht betroffenen Arm. Durch den Spiegel sieht er diese Bewegung als die seiner betroffenen Seite. Erste Studien zeigen eine gewisse Wirksamkeit der Therapie, der genaue Wirkmechanismus ist aber noch unklar.

Holm Thieme ging der Effektivität der Spiegeltherapie hinsichtlich der motorischen Funktionssteigerung in seiner Doktorarbeit nach. Dazu fasste er zunächst die gegenwärtige Evidenz in einer systematischen Übersichtsarbeit, einem Cochrane Review, zusammen. Mit den Daten von 13 der 14 eingeschlossenen randomisierten kontrollierten Studien führte er dann eine Metaanalyse durch. Zusätzlich plante er eine einfach verblindete, randomisierte kontrollierte Studie. Darin untersuchte er die Effektivität der Spiegeltherapie bei Patienten mit einer schweren Armparese im subakuten Stadium nach einem Schlaganfall. In seine Arbeit schloss er $60 \mathrm{~Pa}$ tienten ein und teilte diese in drei Gruppen auf. Zwei Gruppen erhielten über fünf Wochen 20 Einheiten Spiegeltherapie, die jeweils 30 Minuten lang waren - Gruppe A als Einzeltherapie, Gruppe B in einer Gruppe mit maximal sechs Personen. Die dritte Gruppe bekam ein unspezifisches mentales Training, bei dem die reflektierende Spiegelseite nicht zum paretischen Arm zeigte. Dadurch schaltete Holm Thieme den vermuteten Effekt der visuellen Rückmeldung aus.

\section{Ergebnisse}

Holm Thieme hat herausgefunden, dass ...

> die in sein Review eingeschlossenen Studien signifikant positive Effekte der Spiegeltherapie auf die motorische Funktion nachweisen konnten, insbesondere verglichen mit einer Scheintherapie (Plexiglasscheibe). Dies gilt - mit limitierter Evidenz - auch für die motorische Funktion nach sechs Monaten und die Aktivitäten des täglichen Lebens (ADL).

> die Spiegeltherapie schmerzreduzierend zu wirken scheint - besonders bei Patienten mit einem komplexen regionalen Schmerzsyndrom nach Schlaganfall.

> sich in seiner Therapiestudie die Ergebnisse aller drei Gruppen hinsichtlich Motorik, Sensorik, ADLs und Lebensqualität nicht unterschieden.

> die Spiegeltherapie jedoch einen visuellräumlichen Neglekt effektiver reduziert als das Mentaltraining.

\section{Fazit}

Zusammenfassend kann Holm Thieme festhalten, dass ...

> die Spiegeltherapie als Zusatzintervention zur Verbesserung der motorischen Funktion der oberen Extremität empfehlenswert ist.

> sich die Spiegeltherapie auch als Gruppenintervention für Patienten mit schwerer Armparese eignet.

$>$ noch nicht abschließend einzuschätzen ist, welche Faktoren (etwa Dosis, Frequenz und Dauer) die Effektivität der Spiegeltherapie beeinflussen.

Eva Trompetter

$\Rightarrow$ Thieme H. Die Spiegeltherapie zur Steigerung der motorischen Funktionen nach Schlaganfall. Dissertation an der Medizinischen Fakultät der Martin-Luther-Universität Halle-Wittenberg; 2012 